\title{
Comprehensive Interpretation of a Three-Point Gauss Quadrature with Variable Sampling Points and Its Application to Integration for Discrete Data
}

\author{
Young-Doo Kwon, ${ }^{1}$ Soon-Bum Kwon, ${ }^{1}$ Bo-Kyung Shim, ${ }^{2}$ and Hyun-Wook Kwon ${ }^{3}$ \\ ${ }^{1}$ School of Mechanical Engineering \& IEDT, Kyungpook National University, Daegu 702-701, Republic of Korea \\ ${ }^{2}$ Department of Mechanical Engineering, Pohang College, Pohang 791-711, Republic of Korea \\ ${ }^{3}$ System Solution Research Department, Research Institute of Industrial Science \& Technology, Pohang 790-600, Republic of Korea \\ Correspondence should be addressed to Hyun-Wook Kwon; kwonhw@rist.re.kr
}

Received 2 September 2013; Revised 27 November 2013; Accepted 28 November 2013

Academic Editor: Vit Dolejsi

Copyright ( 2013 Young-Doo Kwon et al. This is an open access article distributed under the Creative Commons Attribution License, which permits unrestricted use, distribution, and reproduction in any medium, provided the original work is properly cited.

\begin{abstract}
This study examined the characteristics of a variable three-point Gauss quadrature using a variable set of weighting factors and corresponding optimal sampling points. The major findings were as follows. The one-point, two-point, and three-point Gauss quadratures that adopt the Legendre sampling points and the well-known Simpson's 1/3 rule were found to be special cases of the variable three-point Gauss quadrature. In addition, the three-point Gauss quadrature may have out-of-domain sampling points beyond the domain end points. By applying the quadratically extrapolated integrals and nonlinearity index, the accuracy of the integration could be increased significantly for evenly acquired data, which is popular with modern sophisticated digital data acquisition systems, without using higher-order extrapolation polynomials.
\end{abstract}

\section{Introduction}

Numerical integration methods may be grouped in two categories. One is the rule for discrete data and the other is for function of continuous data. The Gauss-Legendre quadrature [1-6] is a well-known rule belonging to the latter category. In the former category for discrete data, the Newton-Cotes [79] method is applicable with many orders of integration. The 1st-order rule is the same as the trapezoidal rule [7] which can be extended to the Romberg integration method [7]. The 2ndorder rule is the same as Simpson's 1/3rd rule, and 3rd-order rule is the same as Simpson's $3 / 8$ th rule [7]. The 4 th-order rule of Newton-Cotes is known as Boole's rule using 5 data points [7]. As the order of integration in the Newton-Cotes methods increases, Runge's phenomenon arises and the accuracy of integration becomes worse owing to the fluctuation of the interpolated higher-order polynomials [10].

The Gauss-Legendre quadrature uses function values at interior sampling points with corresponding best weights to result in a very accurate result in spite of the relatively small number of sampling points. However, this quadrature is inapplicable to discrete data points because it does not use boundary point data. In this study, we provide a lemma with a formula for the new 3-point Gauss quadrature of variable sampling points which include the Legendre point as well.

An examination of the effect of these varied sampling points found that the one-point, two-point, and three-point Gauss quadratures adopting the Legendre sampling points and Simpson's 1/3 rule were actually special cases of the variable three-point Gauss quadrature. The order of the polynomial that can be integrated precisely by conventional three-point Gauss quadrature is 5. On the other hand, the use of varied sampling points with a variable three-point Gauss quadrature does not allow exact integration of a polynomial order 5.

Despite their reduced accuracy, variable Gauss quadratures can be applied effectively to special situations, for example, the shear-locking problems that arise when using the finite element method for a plate/shell when the ratio of the 
thickness to the width is quite small, as reported previously $[11,12]$. Another effective application is integration of discrete data, which is the same as Simpson's rule, rather than the conventional Gauss quadrature which is inapplicable owing to its use of inner-range data. For a weighting factor of $\alpha=4 / 3$ at the central sampling point, the optimal sampling positions for the outer sampling points were obtained as end points. Therefore, when using this specific group of weighting factor and sampling points, the end point Gauss quadrature becomes equal to Simpson's $1 / 3$ rule. If the central weighting factor of $\alpha$ is increased further to $22 / 12$ or $52 / 27$, this produces a 1st extended end point integration or 2 nd extended end point integration, where the outer sampling points are located at the extended outer end points \pm 2 or \pm 3 .

Accordingly, this study examined the characteristics of various groups of weighting factors and sampling points and tested the performance of the extended end points quadrature using the outer out-of-domain sampling points. A new method, adopting quadratically extrapolated integrals and a nonlinearity index, using integrals of the variable threepoint Gauss integrations of the 1st and 2nd extended end points and conventional end point integration, was applied to the integration of evenly acquired discrete data to obtain new four kinds of numerical integration formulae.

\section{Variable Three-Point Gauss Integration Formula with Variable Weighting Factors and Sampling Points}

The modification of the Gauss integration formula with a near-zero center-weight factor was included in a previous study [11]. This paper presents a comprehensive interpretation of the variable three-point Gauss integration formula with effective applications. Essentially, to vary the Gauss integration weights, the weighting factors are represented by a central weighting factor $W_{2}=\alpha$, and any changes in the central weighting factor are reflected in the weights and locations of outer sampling points for the optimal integration. The detailed procedure to derive the variable three-point Gauss integration formula with the weighting factors is as follows [11]:

$$
\int_{-1}^{1} f d x=W_{1} f\left(r_{1}\right)+W_{2} f\left(r_{2}\right)+W_{3} f\left(r_{3}\right)
$$

where $r_{1}, r_{2}$, and $r_{3}$ are the sampling points within the range of $-1 \leq r_{i} \leq 1$. In addition, the integration weights $\left(W_{1}, W_{2}\right.$, and $W_{3}$ ) are given by the following expressions:

$$
W_{i}=\int_{-1}^{1} l_{i}(r) d r
$$

where $l_{i}(r)$ is the Legendre polynomial formula,

$$
l_{i}(r)=\frac{\left(r-r_{1}\right)\left(r-r_{2}\right)\left(r-r_{3}\right)}{\left(r_{i}-r_{1}\right)\left(r_{i}-r_{2}\right)\left(r_{i}-r_{3}\right)}
$$

The general expressions for the sampling points and weights of the Gauss integration can be driven as follows. In (2), if it is assumed that the weight $W_{2}=\alpha$ at $r_{2}=0$ and the other weights are $W_{1}, W_{3}$ at $r_{1}=-\xi$ and $r_{3}=\xi$, respectively, then the optimum results can be obtained as follows:

$$
\begin{aligned}
W_{2} & =\int_{-1}^{1} l_{2}(r) d r=\int_{-1}^{1} \frac{\left(r-r_{1}\right)\left(r-r_{3}\right)}{\left(r_{2}-r_{1}\right)\left(r_{2}-r_{3}\right)} d r \\
& =\int_{-1}^{1} \frac{(r+\xi)(r-\xi)}{(0+\xi)(0-\xi)} d r \\
& =\int_{-1}^{1} \frac{r^{2}-\xi^{2}}{-\xi^{2}} d r=-\frac{2}{3 \xi^{2}}+2=\alpha .
\end{aligned}
$$

From (4), the relationship between the central weighting factor $\alpha$ and the corresponding optimal location of outer sampling point is as follows:

$$
r_{3}=-r_{1}=\xi=\sqrt{\frac{2}{3(2-\alpha)}} .
$$

We present a lemma regarding three-point Gauss quadrature of variable sampling point.

Lemma 1. Integral I from -1 to 1 of function $f$ is obtained numerically by the equation

$$
I=W_{1} f\left(r_{1}\right)+W_{2} f\left(r_{2}\right)+W_{3} f\left(r_{3}\right),
$$

where

$$
\begin{gathered}
W_{1}=1-\frac{\alpha}{2}, \quad \text { at } r_{1}=-\xi, \\
W_{2}=\alpha, \quad \text { at } r_{2}=0, \\
W_{3}=1-\frac{\alpha}{2}, \quad \text { at } r_{3}=\xi=\sqrt{\frac{2}{3(2-\alpha)}},
\end{gathered}
$$

which is exact for the polynomial of order up to 3.

Proof. Without loss of generality, we assumed the range of integration $r$, which is a mapped variable, from -1 to 1 . For the odd monomials with odd index such as $x^{1}$ and $x^{3}$, the integrals are 0 obviously. For the constant integrand $c$, the integral is exactly $2 c$, because sum of the weights is 2 . We only need to verify that the integral of the integrand $x^{2}$ is $2 / 3$.

Equation (6) for the integrand of $x^{2}$ is

$$
\begin{aligned}
I & =W_{1} f\left(r_{1}\right)+W_{2} f\left(r_{2}\right)+W_{3} f\left(r_{3}\right) \\
& =\frac{(2-\alpha) \xi_{1}^{2}}{2}+\alpha \xi_{2}^{2}+\frac{(2-\alpha) \xi_{3}^{2}}{2}=(2-\alpha) \xi^{2}=\frac{2}{3} .
\end{aligned}
$$

Equation (7) shows the general expression for the sampling points and weights of the variable three-point Gauss integration rule. The weighting factor $\alpha\left(=W_{2}\right)$ can be selected arbitrarily within the range of $0 \sim 2$. 
TABle 1: Different types of 3-point Gauss integration of variable sampling points and the integration characteristics.

\begin{tabular}{|c|c|c|c|c|c|}
\hline Type & $\begin{array}{l}\text { Weight } \\
\text { ratio } \varphi \\
\left(=W_{2} / W_{1}\right)\end{array}$ & $\begin{array}{l}\text { Weighting } \\
\text { factor } \alpha\end{array}$ & $\begin{array}{l}\text { Integration } \\
\text { weights }\end{array}$ & $\begin{array}{l}\text { Sampling } \\
\text { points }\end{array}$ & Integration characteristics \\
\hline (a) & 0 & 0.0 & $\begin{array}{l}1.00000000 \\
0.00000000\end{array}$ & $\begin{array}{c} \pm 0.57735027 \\
0.00000000\end{array}$ & $\begin{array}{l}\text { Conventional two-point rule } \\
\text { (Legendre sampling points) }\end{array}$ \\
\hline (b) & $\varepsilon$ & $1 \times 10^{-4}$ & $\begin{array}{l}0.99995000 \\
0.00010000\end{array}$ & $\begin{array}{c} \pm 0.57736470 \\
0.00000000\end{array}$ & $\begin{array}{l}\text { Quasi two-point rule } \\
\text { (near-zero center-weight } \\
\text { three-point rule) }\end{array}$ \\
\hline (c) & 1 & $2 / 3$ & $\begin{array}{l}0.66666667 \\
0.66666667\end{array}$ & $\begin{array}{c} \pm 0.70710678 \\
0.00000000\end{array}$ & $\begin{array}{l}\text { Three-point rule } \\
\text { (even weight) }\end{array}$ \\
\hline (d) & $8 / 5$ & $8 / 9$ & $\begin{array}{l}0.55555556 \\
0.88888889\end{array}$ & $\begin{array}{c} \pm 0.77459667 \\
0.00000000\end{array}$ & $\begin{array}{l}\text { Conventional three-point rule } \\
\text { (Legendre sampling points) }\end{array}$ \\
\hline (e) & 2 & 1.0 & $\begin{array}{l}0.50000000 \\
1.00000000\end{array}$ & $\begin{array}{c} \pm 0.81649658 \\
0.00000000\end{array}$ & $\begin{array}{l}\text { Three-point rule } \\
\text { (double-center-weight) }\end{array}$ \\
\hline (f) & 4 & $4 / 3$ & $\begin{array}{l}0.33333333 \\
1.33333333\end{array}$ & $\begin{array}{c} \pm 1.00000000 \\
0.00000000\end{array}$ & $\begin{array}{l}\text { Three-point rule } \\
\text { (end-point rule and Simpson's 1/3 rule) }\end{array}$ \\
\hline (g) & 22 & $22 / 12$ & $\begin{array}{l}0.08333333 \\
1.83333333\end{array}$ & $\begin{array}{c} \pm 2.00000000 \\
0.00000000\end{array}$ & $\begin{array}{l}\text { Three-point rule } \\
\text { (1st extended end-point) }\end{array}$ \\
\hline (h) & 52 & $52 / 27$ & $\begin{array}{l}0.03703704 \\
1.92592592\end{array}$ & $\begin{array}{c} \pm 3.00000000 \\
0.00000000\end{array}$ & $\begin{array}{c}\text { Three-point rule } \\
\text { (2nd extended end-point) }\end{array}$ \\
\hline (i) & $\infty$ & 2.0 & $\begin{array}{l}0.00000000 \\
2.00000000\end{array}$ & $\begin{array}{c}\infty \\
0.00000000\end{array}$ & $\begin{array}{l}\text { Conventional one-point rule } \\
\text { (Legendre sampling point) }\end{array}$ \\
\hline
\end{tabular}

\section{Classification of a Set of Weighting Factors and Sampling Points and Their Integration Characteristics}

In the variable three-point Gauss integration formula, the term of "variable" was adopted because the weighting factor $\alpha$ can be selected arbitrarily within the range of $0 \sim 2$. In addition, any change in the central weighting factor is also reflected in the weighting factors and locations of outer sampling points, thereby changing the numerical integration characteristics ((5) and (7)). These different groups of integration weights and sampling points can be classified according to their weight ratio $\varphi\left(=W_{2} / W_{1}\right)$.

Table 1 lists nine fundamental weight ratios. The type (a) ratio of $\varphi=0$ corresponds to the conventional two-point Gauss-Legendre quadrature, which is a special case of the variable three-point Gauss quadrature. The type (b) ratio of $\varphi=\varepsilon$ corresponds to the quasi two-point rule, which is applied in FEM to prevent numerical instability [11]. The type (c) ratio of $\varphi=1$ corresponds to the even weight threepoint rule that might be applied effectively to some problems in the future. The type (d) ratio of $\varphi=8 / 5$ corresponds to the conventional three-point Gauss-Legendre quadrature. The type (e) ratio of $\varphi=2$ corresponds to the doublecenter-weight three-point rule that might also be applied effectively to some problems in the future. The type (f) ratio of $\varphi=4$ corresponds to the end point rule, which is the same as Simpson's $1 / 3$ rule, where the outer sampling points are located at the end points of a dimensionless domain of $[-1 \sim 1]$. The type $(\mathrm{g})$ ratio of $\varphi=22$ corresponds to the 1st extended end point rule, where the outer sampling points are located at the end points of an extended dimensionless domain of $[-2 \sim 2]$. The type $(\mathrm{h})$ ratio of $\varphi=52$ corresponds to the 2 nd extended end point rule, where the outer sampling points are located at the end points of a doubly extended dimensionless domain of [ $-3 \sim 3]$. Finally, the type (i) ratio of $\varphi=\infty$ corresponds to the extended end point rule, where the outer sampling points are located at the end points of an infinitely extended dimensionless domain of $[-\infty \sim \infty]$, which is actually the conventional one-point Gauss-Legendre quadrature.

The variable three-point rule of a Gauss quadrature includes the conventional Gauss-Legendre quadrature of the one-point rule $(\varphi=\infty)$ and two-point rule $(\varphi=0)$, as well as the three-point rule $(\varphi=8 / 5)$. In addition, it also includes the well-known Simpson's 1/3 rule when $\varphi=4$. This can also be expanded further to the cases of $\varphi=\varepsilon, \varphi=1, \varphi=2, \varphi=22$, and $\varphi=52$, respectively. Each case has particular meaning related to the weighting factors or sampling points. Figure 1 shows the proportional change in the integration weights and sampling points according to the weight ratio.

We present three corollaries regarding three-point Gauss quadrature of variable sampling point.

Corollary 2. The three-point Gauss quadrature of Lemma 1 (6) (7) reduces to one-point Gauss-Legendre quadrature with $\alpha=2$.

Corollary 3. The three-point Gauss quadrature of Lemma 1 (6) (7) reduces to two-point Gauss-Legendre quadrature with $\alpha=0$.

Corollary 4. The three-point Gauss quadrature of Lemma 1 (6) (7) reduces to Simpson's $1 / 3$ rule with $\alpha=4 / 3$. 
TABLE 2: Relative errors of the different types of 3-point Gauss integration of variable sampling pints for 5 monomial integrands.

\begin{tabular}{|c|c|c|c|c|c|}
\hline \multirow{2}{*}{ Weight ratio $(\varphi)$} & \multicolumn{5}{|c|}{ Relative errors for each monomial integrand } \\
\hline & $x^{1}$ & $x^{2}$ & $x^{3}$ & $x^{4}$ & $x^{5}$ \\
\hline 0 & $0.0000 E+00$ & $0.8974 E-08$ & $0.1795 E-07$ & $0.2778 E-01$ & $0.8333 E-01$ \\
\hline$\varepsilon$ & $0.0000 E+00$ & $0.1761 E-07$ & $0.3522 E-07$ & $0.2778 E-01$ & $0.8333 E-01$ \\
\hline 1 & $0.0000 E+00$ & $0.1228 E-07$ & $0.2456 E-07$ & $0.1042 E-01$ & $0.3125 E-01$ \\
\hline $8 / 5$ & $0.0000 E+00$ & $0.1264 E-07$ & $0.2528 E-07$ & $0.3829 E-07$ & $0.5168 E-07$ \\
\hline 2 & $0.0000 E+00$ & $0.1830 E-07$ & $0.3661 E-07$ & $0.6944 E-02$ & $0.2083-E 01$ \\
\hline 4 & $0.0000 E+00$ & $0.1490 E-07$ & $0.2980 E-07$ & $0.4167 E-01$ & $0.1250 E+00$ \\
\hline 22 & $0.0000 E+00$ & $0.1192 E-06$ & $0.2384 E-06$ & $0.3542 E+00$ & $0.1063 E+01$ \\
\hline 52 & $0.0000 E+00$ & $0.2533 E-06$ & $0.5066 E-06$ & $0.8750 E+00$ & $0.2625 E+01$ \\
\hline$\infty$ & $0.0000 E+00$ & $0.2500 E+00$ & $0.5000 E+00$ & $0.6875 E+00$ & $0.8125 E+00$ \\
\hline
\end{tabular}

The italic numbers mean improper results.

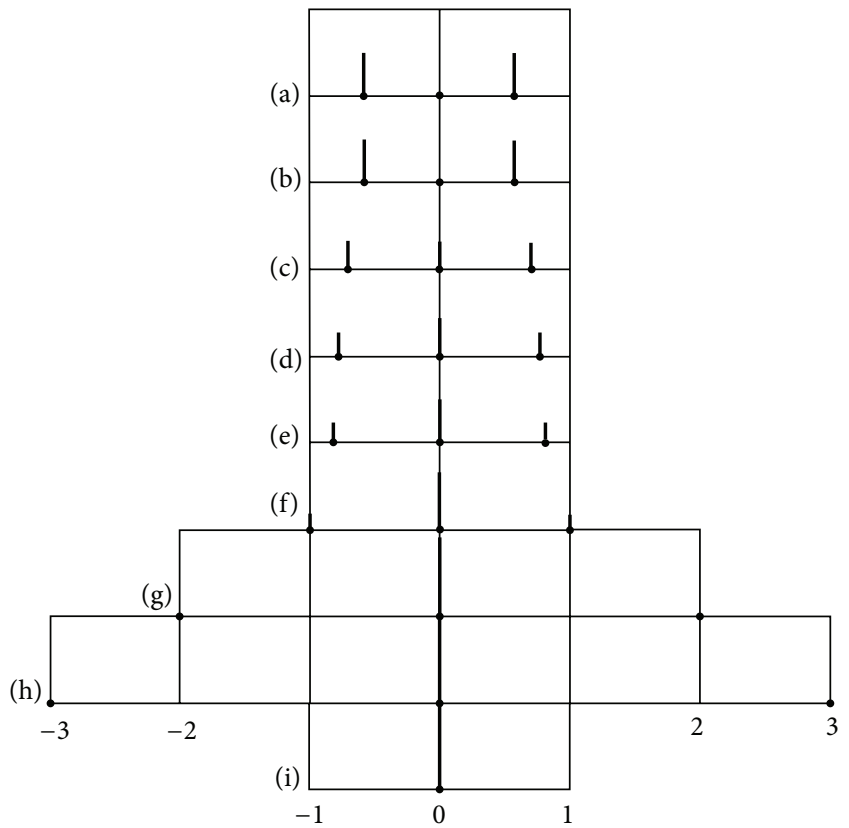

FIGURE 1: Various sets of integration weights and optimal sampling points with respect to the weight ratio $\varphi$; (a) $\varphi=0$, (b) $\varphi=\varepsilon$, (c) $\varphi=1$, (d) $\varphi=8 / 5$, (e) $\varphi=2$, (f) $\varphi=4$, (g) $\varphi=22$, (h) $\varphi=52$, and (i) $\varphi=\infty$.

\section{Extrapolated Quadratic Polynomial of the Integrals Using Data from End Point Integral $(\varphi=4)$ and Extended End$$
\text { Point Integrals }(\varphi=22,52) \text { for }
$$$$
\text { Evenly Acquired Discrete Data }
$$

To test the characteristics of variable three-point Gauss integration formulae, the error ratios of the integral from 0 to 2 for the 5 types of monomial integrand were compared (Table 2). Most types exhibited accurate results up to the cubic monomial except in the case of a weight ratio $\varphi=$ $\infty$ (conventional one-point rule). The type of weight ratio $\varphi=8 / 5$ (conventional three-point rule of Legendre sampling points) results in very accurate result up to the $x^{5}$ monomial, as expected.

According to the formula of Gauss quadrature of variable three sampling points with corresponding best weights, one can see that the sampling points may be located at the boundary points (integral $I_{1}$ ) which means that the method is applicable to discrete data points. Moreover, the outer sampling points may be located out of region such as 2nd next discrete points (integral $I_{2}$ ) and 3rd next discrete points (integral $I_{3}$ ). Using the integrals $I_{1}, I_{2}$, and $I_{3}$, we can extrapolate the integral function $I_{\mathrm{GL}}$, like Richardson's extrapolation to interval $h=0$ [7], to the case of Legendre sampling point of $(0.6)^{1 / 2}$, where there is no data.

In other words, when one integrates a function from $-1 \sim 1$ in local coordinate $\xi$, the best sampling points are the Legendre points of $r_{1}=-r_{3}, r_{2}=0$, and $r_{3}=(0.6)^{1 / 2}$. However, data are only on the discrete points of $r=\ldots-$ $3,-2,-1,0,1,2,3 \ldots$ Thus, we compute the following three integrals first.

$$
I_{1}\left(r_{3}=1\right) \text { : }
$$

weights: $W_{1}=W_{3}, \quad W_{2}=\frac{4}{3}, \quad W_{3}=\frac{\left(2-W_{2}\right)}{2}$,

sampling points: $r_{1}=-r_{3}, \quad r_{2}=0, \quad r_{3}=1$.

$$
I_{2}\left(r_{3}=2\right) \text { : }
$$

weights: $W_{1}=W_{3}, \quad W_{2}=\frac{22}{12}, \quad W_{3}=\frac{\left(2-W_{2}\right)}{2}$,

sampling points: $r_{1}=-r_{3}, \quad r_{2}=0, \quad r_{3}=2$.

$$
I_{3}\left(r_{3}=3\right) \text { : }
$$

weights: $W_{1}=W_{3}, \quad W_{2}=\frac{52}{27}, \quad W_{3}=\frac{\left(2-W_{2}\right)}{2}$,

sampling points: $r_{1}=-r_{3}, \quad r_{2}=0, \quad r_{3}=3$. 
TABLE 3

\begin{tabular}{lcccc}
\hline$a_{-2}$ & $a_{-1}$ & $a_{0}$ & $a_{1}$ & $a_{2}$ \\
\hline$(-1+\sqrt{ } 0.6) / 12$ & $(8-4 \sqrt{ } 0.6) / 12$ & $(10+6 \sqrt{ } 0.6) / 12$ & $(8-4 \sqrt{ } 0.6) / 12$ & $(-1+\sqrt{ } 0.6) / 12$ \\
-0.018783611 & 0.40846778 & 1.2206317 & 0.40846778 & -0.018783611 \\
\hline
\end{tabular}

Then, we extrapolate these integrals to compute the integral $I_{\mathrm{GL}}\left(r_{3}=(0.6)^{1 / 2}\right)$, which cannot be obtained directly as follows because there is no data on the sampling point $r_{3}=(0.6)^{1 / 2}$ :

$$
I_{\mathrm{GL}}\left(r_{3}=(0.6)^{1 / 2}\right) \text { : }
$$

weights: $W_{1}=W_{3}, \quad W_{2}=\frac{8}{9}, \quad W_{3}=\frac{\left(2-W_{2}\right)}{2}$, sampling points: $r_{1}=-r_{3}, \quad r_{2}=0 ., \quad r_{3}=(0.6)^{1 / 2}$.

The process of extrapolation is presented here. The integral, which is a function of outer sampling point $x=r_{3}$, may be treated as a quadratic polynomial and its coefficients are determined through the interpolating points $\left(1, I_{1}\right),\left(2, I_{2}\right)$, and $\left(3, I_{3}\right) . I_{1}=I\{\operatorname{type}(f), x=1\}, I_{2}=I\{\operatorname{type}(g), x=2\}$, and $I_{3}=I\{\operatorname{type}(h), x=3\}$ are given in (9) (11).

On the other hand, the error of the integral choosing the outer sampling point as $x=1,2$, or 3 increases explosively. Therefore, a nonlinearity index, $N$, is used in a quadratically interpolated integral, which is a function of the absolute position of the outer sampling point $z=x^{N}$. Hence, the quadratic polynomial of the integral, depending on the position of the outer sampling point of the three-point Gauss quadrature, can be represented as follows:

$$
I(z)=a_{0}+a_{1} z+a_{2} z^{2},
$$

where $z=$ nonlinearly mapped variable of the absolute position of the outer sampling point $x, I\left[z=1^{N}\right]=I_{1}$ : end point integration $(\varphi=4), I\left[z=2^{N}\right]=I_{2}$ : 1st extended end point integration $(\varphi=22), I\left[z=3^{N}\right]=I_{3}$ : 2nd extended end point integration $(\varphi=52)$, and $N=$ nonlinearity index.

After obtaining the coefficients, $a_{0}, a_{1}$, and $a_{2}$, of the polynomial, the best expected integral can be predicted with the nonlinearly mapped extrapolated outer sampling point $[z=$ $\left.\left(x=(0.6)^{1 / 2}\right)^{N}\right]$, where the point $\left(x=(0.6)^{1 / 2}\right)$ is the Legendre sampling point of the conventional three points of Gauss quadrature, and that point originally could not be sampled for this type of discrete data. This whole procedure can be repeated for the linear interpolation of the integral $I$, where we use only two data of points $\left(1, I_{1}\right)$ and $\left(2, I_{2}\right) . I_{1}=$ $I\{\operatorname{type}(f), x=1\}$ and $I_{2}=I\{\operatorname{type}(g), x=2\}$ are given in (9) (10).

We present four theorems regarding extrapolated Gauss quadrature of extended end points.

Theorem 5. Integral I from -1 to 1 of discrete data $f_{i}$ is obtained numerically by formula $I$,

$$
I=\left\{a_{-2} f_{-2}+a_{-1} f_{-1}+a_{0} f_{0}+a_{1} f_{1}+a_{2} f_{2}\right\},
$$

TABLE 4

\begin{tabular}{lcccc}
\hline$a_{-2}$ & $a_{-1}$ & $a_{0}$ & $a_{1}$ & $a_{2}$ \\
\hline$-1 / 90$ & $34 / 90$ & $114 / 90$ & $34 / 90$ & $-1 / 90$ \\
-0.011111111 & 0.377777778 & 1.2666667 & 0.37777778 & -0.011111111 \\
\hline
\end{tabular}

which is exact for the polynomial of order up to 3 with the nonlinearity index $N=1$ (see Table 3).

Proof. As in the proof of Lemma 1, we only need to verify that the integral of the integrand $x^{2}$ is $2 / 3$.

Equation (14) for the integrand $x^{2}$ is

$$
\begin{aligned}
I= & \left\{a_{-2} f_{-2}+a_{-1} f_{-1}+a_{0} f_{0}+a_{1} f_{1}+a_{2} f_{2}\right\} \\
= & \frac{4(-1+\sqrt{ } 0.6)}{12}+\frac{(8-4 \sqrt{ } 0.6)}{12} \\
& +\frac{(8-4 \sqrt{ } 0.6)}{12}+\frac{4(-1+\sqrt{ } 0.6)}{12}=\frac{2}{3} .
\end{aligned}
$$

Theorem 6. Integral I from -1 to 1 of discrete data $f_{i}$ is obtained numerically by formula II,

$$
I=\left\{a_{-2} f_{-2}+a_{-1} f_{-1}+a_{0} f_{0}+a_{1} f_{1}+a_{2} f_{2}\right\},
$$

which is exact for the polynomial of order up to 5 with the nonlinearity index $N=2$ (see Table 4).

Proof. As in the proof of Theorem 5, we need to verify that the integral of the integrand $x^{2}$ is $2 / 3$ and integral of $x^{4}$ is $2 / 5$.

Equation (16) for the integrand $x^{2}$ is

$$
\begin{aligned}
I & =\left\{a_{-2} f_{-2}+a_{-1} f_{-1}+a_{0} f_{0}+a_{1} f_{1}+a_{2} f_{2}\right\} \\
& =\frac{-4}{90}+\frac{34}{90}+\frac{34}{90}+\frac{-4}{90}=\frac{2}{3} .
\end{aligned}
$$

Equation (16) for the integrand $x^{4}$ is

$$
\begin{aligned}
I & =\left\{a_{-2} f_{-2}+a_{-1} f_{-1}+a_{0} f_{0}+a_{1} f_{1}+a_{2} f_{2}\right\} \\
& =\frac{-16}{90}+\frac{34}{90}+\frac{34}{90}+\frac{-16}{90}=\frac{2}{5} .
\end{aligned}
$$

Theorem 7. Integral I from -1 to 1 of discrete data $f_{i}$ is obtained numerically by formula III,

$$
I=\left\{a_{-3} f_{-3}+a_{-2} f_{-2}+a_{-1} f_{-1}+a_{0} f_{0}+a_{1} f_{1}+a_{2} f_{2}+a_{3} f_{3}\right\},
$$

which is exact for the polynomial of order up to 5 with the nonlinearity index $N=1$ (see Table 5). 
TABLE 5

\begin{tabular}{lcccccc}
\hline$a_{-3}$ & $a_{-2}$ & $a_{-1}$ & $a_{0}$ & $a_{1}$ & $a_{2}$ & $a_{3}$ \\
\hline$(1.3-1.5 \sqrt{ } 0.6) / 27$ & $(-3.6+4 \sqrt{ } 0.6) / 12$ & $(3.3-2.5 \sqrt{ } 0.6) / 3$ & $41 / 135+10 \sqrt{ } 0.6 / 9$ & $(3.3-2.5 \sqrt{ } 0.6) / 3$ & $(-3.6+4 \sqrt{ } 0.6) / 12$ & $(1.3-1.5 \sqrt{ } 0.6) / 27$ \\
0.0051149999 & -0.041801110 & 0.45450278 & 1.1643667 & 0.45450278 & -0.041801110 & 0.0051149999 \\
\hline
\end{tabular}

TABLE 6

\begin{tabular}{|c|c|c|c|c|c|c|}
\hline$a_{-3}$ & $a_{-2}$ & $a_{-1}$ & $a_{0}$ & $a_{1}$ & $a_{2}$ & $a_{3}$ \\
\hline $16.32 / 12960$ & $-241.92 / 12960$ & $5140.80 / 12960$ & $16089.60 / 12960$ & $5140.80 / 12960$ & $-241.92 / 12960$ & $16.32 / 12960$ \\
\hline 0.0012592593 & -0.018666667 & 0.39666667 & 1.2414815 & 0.39666667 & -0.018666667 & 0.0012592593 \\
\hline
\end{tabular}

Proof. As in the proof of Theorem 6, we need to verify that the integral of the integrand $x^{2}$ is $2 / 3$ and integral of $x^{4}$ is $2 / 5$. Equation (19) for the integrand $x^{2}$ is

$$
\begin{aligned}
& I=\left\{a_{-3} f_{-3}+a_{-2} f_{-2}+a_{-1} f_{-1}+a_{0} f_{0}\right. \\
&\left.+a_{1} f_{1}+a_{2} f_{2}+a_{3} f_{3}\right\}=\frac{9(1.3-1.5 \sqrt{ } 0.6)}{27} \\
&+\frac{4(-3.6+4 \sqrt{ } 0.6)}{12}+\frac{(3.3-2.5 \sqrt{ } 0.6)}{3} \\
&+\frac{(3.3-2.5 \sqrt{ } 0.6)}{3}+\frac{4(-3.6+4 \sqrt{ } 0.6)}{12} \\
&+\frac{9(1.3-1.5 \sqrt{0.6})}{27}=\frac{2}{3} .
\end{aligned}
$$

Equation (19) for the integrand $x^{4}$ is

$$
\begin{aligned}
& I=\left\{a_{-3} f_{-3}+a_{-2} f_{-2}+a_{-1} f_{-1}+a_{0} f_{0}\right. \\
&\left.+a_{1} f_{1}+a_{2} f_{2}+a_{3} f_{3}\right\}=\frac{81(1.3-1.5 \sqrt{ } 0.6)}{27} \\
&+\frac{16(-3.6+4 \sqrt{ } 0.6)}{12}+\frac{1(3.3-2.5 \sqrt{ } 0.6)}{3} \\
&+\frac{1(3.3-2.5 \sqrt{ } 0.6)}{3}+\frac{16(-3.6+4 \sqrt{ } 0.6)}{12} \\
&+\frac{81(1.3-1.5 \sqrt{0.6})}{27}=\frac{2}{5} .
\end{aligned}
$$

Theorem 8. Integral I from -1 to 1 of discrete data $f_{i}$ is obtained numerically by formula $I V$,

$$
I=\left\{a_{-3} f_{-3}+a_{-2} f_{-2}+a_{-1} f_{-1}+a_{0} f_{0}+a_{1} f_{1}+a_{2} f_{2}+a_{3} f_{3}\right\},
$$

which is exact for the polynomial of order up to 5 with the nonlinearity index $N=2$ (see Table 6).

As in the proof of Theorem 7, we need to verify that the integral of the integrand $x^{2}$ is $2 / 3$ and integral of $x^{4}$ is $2 / 5$.
Equation (22) for the integrand $x^{2}$ is

$$
\begin{aligned}
I=\left\{a_{-3} f_{-3}+a_{-2} f_{-2}+a_{-1} f_{-1}+a_{0} f_{0}\right. \\
\left.\quad+a_{1} f_{1}+a_{2} f_{2}+a_{3} f_{3}\right\}=\frac{9 * 16.32}{12960} \\
-\frac{4 * 241.92}{12960}+\frac{5140.80}{12960}+\frac{5140.80}{12960} \\
-\frac{4 * 241.92}{12960}+\frac{9 * 16.32}{12960}=\frac{2}{3} .
\end{aligned}
$$

Equation (22) for the integrand $x^{4}$ is

$$
\begin{aligned}
& I=\left\{a_{-3} f_{-3}+a_{-2} f_{-2}+a_{-1} f_{-1}+a_{0} f_{0}\right. \\
&\left.+a_{1} f_{1}+a_{2} f_{2}+a_{3} f_{3}\right\}=\frac{81 * 16.32}{12960} \\
&-\frac{16 * 241.92}{12960}+\frac{5140.80}{12960} \\
&+\frac{5140.80}{12960}-\frac{16 * 241.92}{12960} \\
&+\frac{81 * 16.32}{12960}=\frac{2}{5} .
\end{aligned}
$$

Through this extrapolation to obtain the integral $I_{\mathrm{GL}}\left\{\xi_{3}=\right.$ $\left.\left((0.6)^{1 / 2}\right)^{N=2}=0.6\right\}$, we can integrate polynomial up to the order of 5 exactly and up to the order of 7 approximately, without any fluctuating phenomenon of Runge [10].

We compared the four formulae with Simpson's 1/3 rule for integration of the discrete data from 1 to 3 . The discrete data are given at integer point of $x$ from the monomials $x^{3}$, $x^{7}$, and $\exp (x)$, respectively, as shown in Table 7 .

Table 8 compares the four formulae with Boole's rule for integration of the discrete data from 1 to 5 . The discrete data are given at integer points of $x$ from the monomials $x^{5}, x^{7}$, and $\exp (x)$, respectively. Formula I results in some error for the integrand of $x^{5}$, because it is for the polynomial of order up to 3. Formula II and formula IV still give better result compared with Boole's. 
TABLE 7: Comparison of the integrals using four formulae of extrapolation methods (end-point Gauss quadrature) with Simpson's 1/3 rule.

\begin{tabular}{lccccccc}
\hline Integrand & Error & Exact & Simpson & Formula I & Formula II & Formula III & Formula IV \\
\hline \multirow{3}{*}{$x^{3}$} & Value & $0.200000 E+2$ & $0.200000 E+2$ & $0.200000 E+2$ & $0.200000 E+2$ & $0.200000 E+2$ & $0.200000 E+2$ \\
& $\%$ & 0.0 & 0.0 & 0.0 & 0.0 & 0.0 & - \\
\hline \multirow{3}{*}{$x^{7}$} & Ratio & - & - & - & - & 0.0 & - \\
& Value & $0.820000 E+3$ & $0.900000 E+3$ & $0.742218 E+3$ & $0.806667 E+3$ & $0.858226 E+3$ & $0.819360 E+3$ \\
& $\%$ & 0 & $0.975610 E+1$ & $0.948565 E+1$ & $0.162602 E+1$ & $0.466169 E+1$ & $0.780488 E-1$ \\
& Ratio & - & 1.0 & 0.97 & 0.17 & 0.48 & 0.0080 \\
\multirow{2}{*}{$\operatorname{Exp}(x)$} & Value & $0.173673 E+2$ & $0.174533 E+2$ & $0.172896 E+2$ & $0.173665 E+2$ & $0.174049 E+2$ & $0.173684 E+2$ \\
& $\%$ & & $0.495718 E+0$ & $0.447095 E+0$ & $0.619861 E-1$ & $0.216874 E+0$ & $0.666631 E-2$ \\
& Ratio & - & 1.0 & 0.90 & 0.13 & 0.44 & 0.013 \\
\hline
\end{tabular}

TABLE 8: Comparison of the integrals using four formulae of extrapolation methods (end-point Gauss quadrature) with Boole’s rule.

\begin{tabular}{lccccccc}
\hline Integrand & Error & Exact & Boole & Formula I & Formula II & Formula III & Formula IV \\
\hline \multirow{4}{*}{$x^{5}$} & Value & $0.260400 E+4$ & $0.260400 E+4$ & $0.259848 E+4$ & $0.260400 E+4$ & $0.260400 E+4$ & $0.260400 E+4$ \\
& $\%$ & 0.0 & 0.0 & $0.212143 E+0$ & 0.0 & 0.0 & - \\
\hline \multirow{2}{*}{$x^{7}$} & Ratio & - & - & $\infty$ & - & 0.0 \\
& Value & $0.488280 E+5$ & $0.489560 E+5$ & $0.482853 E+5$ & $0.487880 E+5$ & $0.489427 E+5$ & $0.488261 E+5$ \\
& $\%$ & 0 & $0.262145 E+0$ & $0.111146 E+1$ & $0.819202 E-1$ & $0.234860 E+0$ & $0.393217 E-2$ \\
& Ratio & - & 1.0 & 4.2 & 0.31 & 0.90 & 0.015 \\
\hline \multirow{2}{*}{$\operatorname{Exp}(x)$} & Value & $0.145695 E+3$ & $0.145891 E+3$ & $0.145043 E+3$ & $0.145605 E+3$ & $0.146011 E+3$ & $0.145705 E+3$ \\
& $\%$ & & $0.134295 E+0$ & $0.447095 E+0$ & $0.619861 E-1$ & $0.216874 E+0$ & $0.666631 E-2$ \\
& Ratio & - & 1.0 & 3.3 & 0.46 & 1.6 & 0.050 \\
\hline
\end{tabular}

\section{Conclusion}

(1) A lemma for the variable three-point Gauss quadrature has been presented. Based on that, comprehensive sets of weighting factors and corresponding optimal sampling points were presented.

(2) Three corollaries are presented to show that one-point and two-point Gauss-Legendre quadrature and Simpson's $1 / 3$ rule are special cases of the variable three-point Gauss quadrature.

(3) The variable three-point Gauss quadrature can be applied to end point integration of discrete data, such as Simpson's rule, and even to the 1st and 2nd extended end point integrations that use out of region discrete data.

(4) Four theorems of extrapolation method with nonlinearity index $(N=1$ or 2$)$ are presented. The integrals of variable three-point Gauss integrations of the end point integration and extended end point integration are extrapolated to predict the value at dimensionless $\sqrt{ } 0.6^{N}$ point.

(5) Formula II and formula IV given in the theorems exhibit excellent accuracies compared with Simpson's rule or Boole's rule. The formulae can be applied effectively to evenly acquired data, which is the case in modern sophisticated digital data acquisition systems, without using higher-order extrapolation polynomials which might result in numerical instability.

\section{Acknowledgment}

This research was supported by the Basic Science Research Program through the National Research Foundation of
Korea (NRF) funded by the Ministry of Education (NRF2012R1A1A2008903).

\section{References}

[1] S. C. Chapra and R. P. Canale, Numerical Methods for Engineers, McGraw-Hill, New York, NY, USA, 5th edition, 2006.

[2] D. Elliott, P. R. Johnston, and B. M. Johnston, "Estimates of the error in GaussLegendre quadrature for double integrals," Journal of Computational and Applied Mathematics, vol. 236, no. 6, pp. 1552-1561, 2011.

[3] B. A. Hagler, "D-fold Hermite-Gauss quadrature," Journal of Computational and Applied Mathematics, vol. 136, no. 1-2, pp. 53-72, 2001.

[4] G. Dasgupta, "Integration within polygonal finite elements," Journal of Aerospace Engineering, vol. 16, no. 1, pp. 9-18, 2003.

[5] H. T. Rathod, K. V. Nagaraja, B. Venkatesudu, and N. L. Ramesh, "Gauss Legendre quadrature over a triangle," Journal of the Indian Institute of Science, vol. 84, no. 5, pp. 183-188, 2004.

[6] E. Babolian, M. Masjed-Jamei, and M. R. Eslahchi, "On numerical improvement of Gauss-Legendre quadrature rules," Applied Mathematics and Computation, vol. 160, no. 3, pp. 779-789, 2005.

[7] C. F. Gerald and P. O. Wheatley, Applied Numerical Analysis, Addison-Wesley Longman, Boston, Mass, USA, 1999.

[8] C. O. E. Burg, "Derivative-based closed Newton-Cotes numerical quadrature," Applied Mathematics and Computation, vol. 218, no. 13, pp. 7052-7065, 2012.

[9] W. Zhao and H. Li, "Midpoint derivative-based closed NewtonCotes quadrature," Abstract and Applied Analysis, vol. 2013, Article ID 492507, 10 pages, 2013. 
[10] http://en.wikipedia.org/wiki/Runge's_phenomenon.

[11] H. C. Huang, "Implementation of assumed strain degenerated shell elements," Computers and Structures, vol. 25, no. 1, pp. 147155, 1987.

[12] Y. D. Kwon, N. S. Goo, and B. S. Lim, "Resolution of defects in degenerated shell elements through modification of Gauss integration," International Journal of Modern Physics B, vol. 17, no. 8-9, pp. 1877-1883, 2003. 


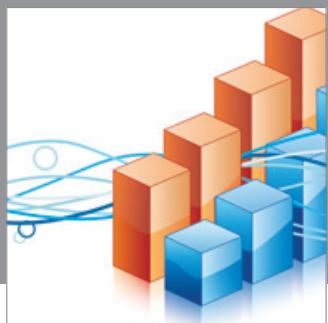

Advances in

Operations Research

mansans

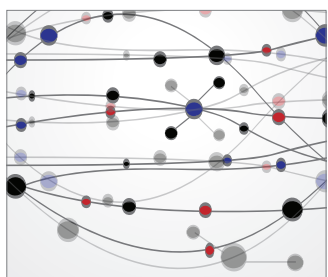

The Scientific World Journal
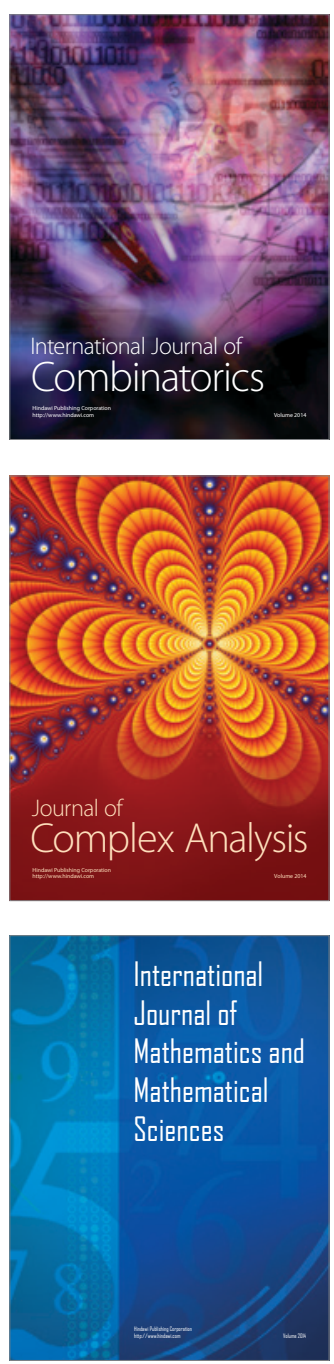
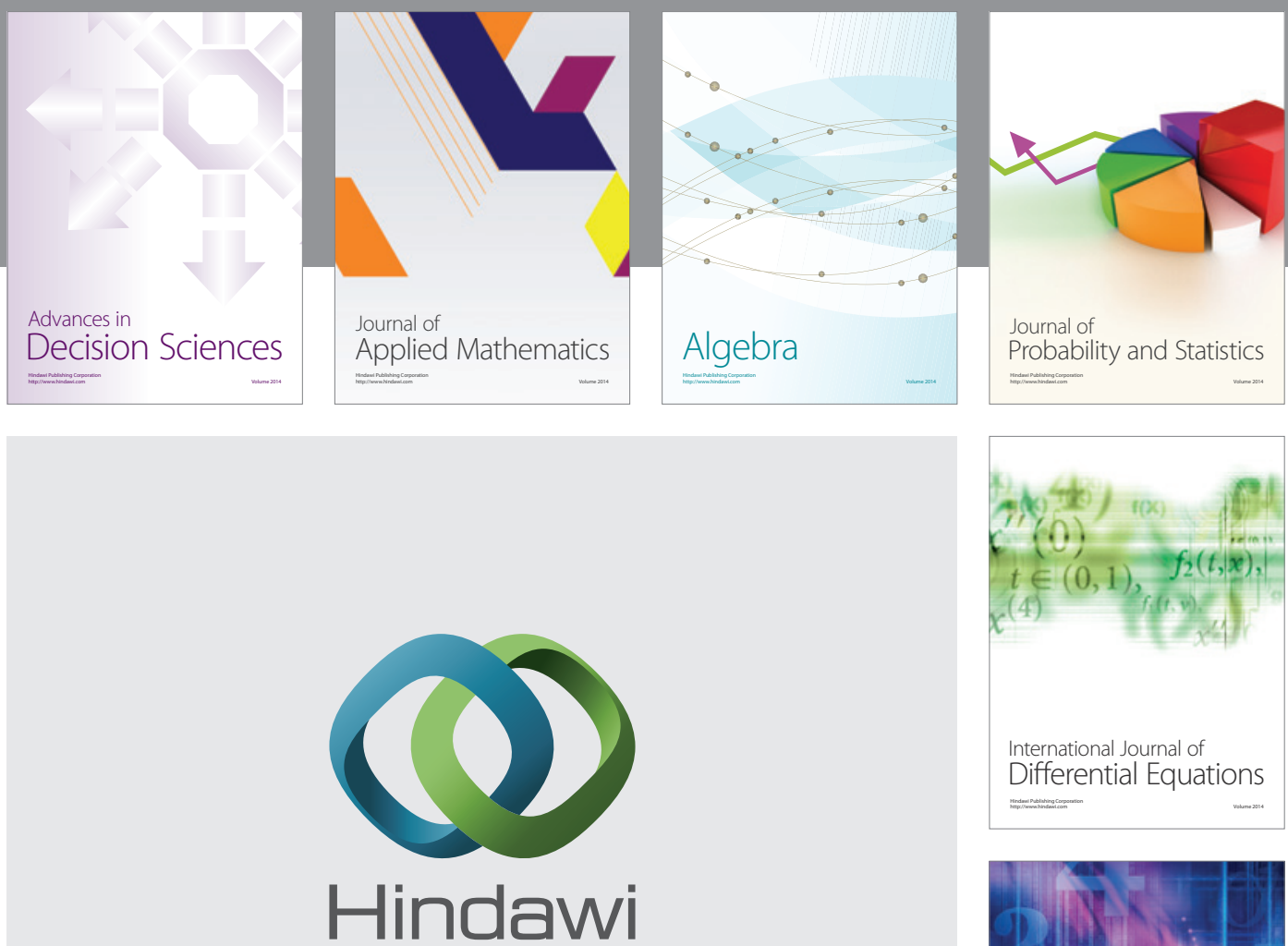

Submit your manuscripts at http://www.hindawi.com
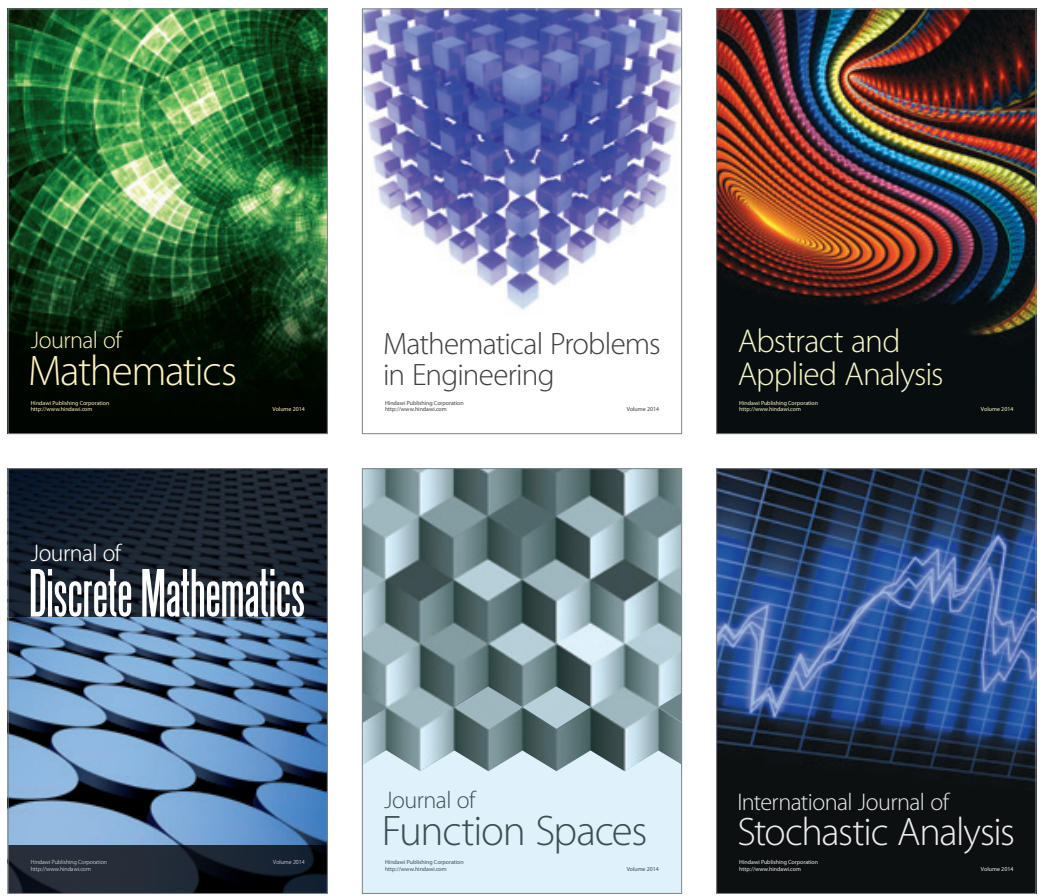

Journal of

Function Spaces

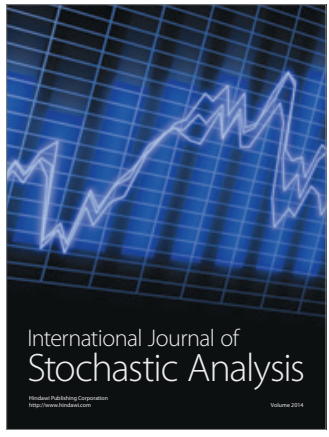

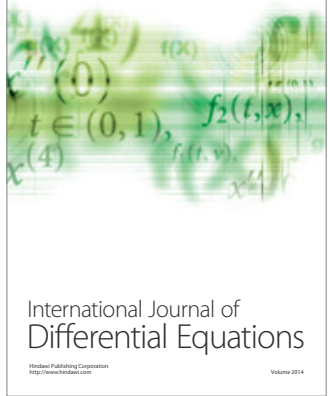
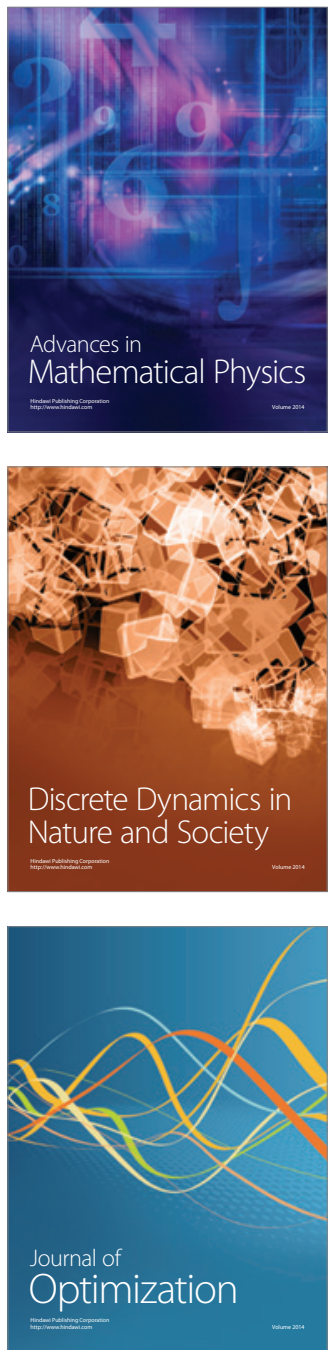